\title{
A rapid detection for the inhibition of phosphoglucose isomerase from Escherichia coli by mercury(II) chloride based on TLC-autographic analysis - preliminary studies
}

\author{
Katarzyna Paradowska $^{1 *}$, Joanna LuteK ${ }^{2}$, Grazyna Ginalska ${ }^{1}$ \\ ${ }^{1}$ Chair and Department of Biochemistry and Biotechnology, Medical University of Lublin, Poland \\ ${ }^{2}$ Student of Chair and Department of Biochemistry and Biotechnology, Medical University of Lublin, Poland
}

\section{ARTICLE INFO \\ Received 21 February 2014 \\ Accepted 11 August 2014}

\section{Keywords:}

TLC-autography,

phosphoglucose isomerase,

inhibitor,

E. coli ATCC 25922,

biofilm.

\begin{abstract}
The quest for new techniques for screening inhibitors of phosphoglucose isomerase is crucially important owing to therapeutic control of chronic bacterial infections associated with the biosynthesis of bacterial biofilm. According to the new method, yellowish zones against the purple background could be visually observed where phosphoglucose isomerase activity was inhibited. The new protocol with NADPH/NBT/PMS staining for TLC-autographic method was able to detect PGI inhibition by pure reference substance as mercury(II) chloride.
\end{abstract}

\section{INTRODUCTION}

Thin Layer Chromatography (TLC)-bioautography analysis is a primary method of detecting anti-microbial compounds from e.g. plant extracts chromatographed on a TLC plates $[15,22]$. To be precise, the bioautographic methods combine chromatographic separation and in situ determination of inhibition microorganism growth $[5,10]$. Three different approaches have been described to screening of antimicrobial compounds on TLC chromatogram [21]. Literature survey has characterized contact, direct and immersion bioautography $[5,6,15]$.

In the contact chromatography (agar diffusion), antimicrobial compounds from the TLC plate are placed by a diffusion on agar inoculated with the microorganisms $[6,16]$. The source of problems in this type of bioautography might be differential diffusion of water insoluble samples from the chromatogram to the agar plate $[6,15]$.

In direct bioautography, TLC layers are sprayed with, or immersed into suspensions of the microorganisms growing in the suitable broth $[15,16]$. In 2D- TLC direct bioautography technique, TLC layers are developed by two solvent systems with very different polarities [6]. The direct bioautographic method requires a lot of attention during manipulations with pathogenic microorganism [15].

\footnotetext{
Corresponding author

e-mail: katarzyna.paradowska@umlub.pl

tel./fax: + 4881 742-37-76
}

All stages of this type of bioautography are performed directly on the plate [16].

In the immersion (agar overlay) bioautography, the chromatogram is covered with a molten, seeded agar medium $[6,16]$. This method is a hybrid between the contact and the direct bioautography, because the tested compounds are transfered by a diffusion from TLC plate to an agar layer as in the contact assay, but the agar layer stays on the plate during the steps: incubation and visualisation as in direct bioautography $[5,8,15]$.

Various methods of visualization of the TLC-bioautograms are available. When working with colored colonies of microorganism, in the presence of the antimicrobial compounds, the inhibition zone of growth is directly visible [8]. When the colonies lack color, tetrazolium salts are usually used as a dehydrogenase activity-detecting compounds $[6,15,17]$. The absence of coloration on a formazan-colored background reveals the presence of antimicrobial agents [8]. It has been reported that some natural compounds e.g. genistein might interfere in the staining with tetrazolium salts through their intensifying reduction [17]. For this reason, another visualization method is proposed. The alternative detection of the bioautogram is associated with the measurement of the light emitted by natural (e.g. Vibrio fischeri) and transgenic luminescent microorganisms [17]. After chromatographic analysis, bioactive compounds are identified as dark spots/zones on a luminescent background [6].

The term TLC-autography is used generally for the application of chemical method in identification of biological effect or process, e.g. activity of free radical scavengers 
or oxidants [15]. A group of the most widely used assays for the detection of radical scavenging and antioxidant properties after TLC include inhibition of bleaching $\beta$-carotene, inhibition of bleaching $\beta$-carotene induced by autooxidation of linoleic acid and tests with 2,2-diphenyl1-picrylhydrazyl (DPPH) or 2,2'-azino-bis(3-ethylbenzthiazoline-6-sulfonic acid (ABTS) reagents $[5,6,15]$.

Also, TLC-autography might be focused on the screening of inhibitors of various enzymes. This method has been reported for screening of acetylcholinesterase $[1,2,4,14,18]$, aldehyde dehydrogenase [13], cyclooxygenase-2 [7], glucosidase [19,24,25], lipase [10], monoamine oxidase [12] and xanthine oxidase [22],

Phosphoglucose isomerase (D-glucose-6-phosphate aldose ketose-isomerase; PGI; EC 5.3.1.9) is an enzyme that plays an important role in the process of bacterial biofilm synthesis. PGI plays a crucial role in the biosynthesis of extracellular polysaccharides (EPS), the compound of microbial biofilm. Therefore, there is huge need to search for novel and fast methods for detecting of PGI inhibitors. Therefore, the principal aim of the present study was to investigate usefulness of TLC-autographic analysis for the determination of phosphoglucose isomerase inhibitors.

\section{MATERIALS AND METHODS}

Bacterial strain. Liquid culture of Escherichia coli (E. coli) reference strain ATCC 25922 was prepared as previously described [20].

Reagents. Bovine serum albumin (BSA), $\beta$-nicotinoamide adenine dinucleotide phosphate $\left(\beta-\mathrm{NADP}^{+}\right)$, dithiotreitol (DTT), fructose 6-phosphate (fru-6P), glucose 6-phosphate (glc-6P), glucose 6-phosphate dehydrogenase from Leuconostoc mesenteroides (L. mesenteroides), morpholinopropane sulfonic acid (MOPS), nitroblue tetrazolium chloride (NBT), phenazine methosulfate (PMS) and tris(hydroxylmethyl)aminomethan (TRIS) were purchased from Sigma-Aldrich (St. Louis, MO, USA). Mercury(II) chloride $\left(\mathrm{HgCl}_{2}\right)$ and ethanol $(\mathrm{EtOH})$ were obtained from $\mathrm{POCH}$ (Gliwice, Poland). Cellulose TLC layers (Merck, Darmstad, Germany) were kindly provided by Department of Physical Chemistry, Medical University of Lublin, Poland. Diaflo YM-100 (cutoff, $100 \mathrm{kDa}$ ) ultrafiltration membranes were received from Millipore Corp. (Bedford, USA).

Spectrophotometric assay for PGI activity. Phosphoglucose isomerase activity was measured using spectrophotometer (Double beam spectrophotometer Evolution 300 UV-VIS, Thermo Fisher Scientific, Madison, USA) and operated on the kinetic mode. The determination of biocatalyst activity was conducted by a coupled assay with glc-6P dehydrogenase from $L$. mesenteroides as auxiliary enzyme at room temperature (RT) according to the method developed by Paradowska et al. [20]. One unit (U) of PGI activity was defined as the amount of enzyme catalyzing $1 \mu \mathrm{mol}$ of $\mathrm{NADP}^{+}$reduction per min.

Partial purification of PGI activity. The characterized enzyme was isolated according to methods described by Paradowska et al. [20] with three minor modifications. Briefly, the purification procedure of phosphoglucose isomerase from E. coli ATCC 25922 included few steps: the culture of microorganism, preparation of crude extract, ammonium sulfate fractionation and ion-exchange chromatography on DEAE-cellulose. Modifications mentioned were conducted during the step of liquid chromatography. Firstly, the PGI activity by spectrophotometric method instead of direct assay for fru-6P with p-hydroxybenzoic acid hydrazide (PAHBAH) was determined. Secondly, the proteins concentration was determined using spectrophotometer (Double beam spectrophotometer Evolution 300 UV-VIS, Thermo Fisher Scientific, Madison, USA) and operated on Warburg-Christian mode [26]. The assays were conducted in the quartz cuvettes. Finally, the most active fractions were collected and desalted by ultrafiltration on the Amicon filtration apparatus (Millipore, Billarica, USA) using YM-100 membrane (instead YM-30 kDa membrane).

TLC-autographic analysis. The assay was preceded by PGI dialysis in order to remove DTT which plays protective function by stabilization of - SH groups of Cys in the active site of the characterized enzyme. Partially purified phosphoglucose isomerase (after ion-exchange chromatography) was dialyzed against $25 \mathrm{mM}$ MOPS buffer, $\mathrm{pH} 7.5$ for 1 hour at $4^{\circ} \mathrm{C}$. Enzymic activity was stabilized by adding BSA at final concentration $0.1 \mathrm{mg} / \mathrm{ml}$ during dialysis. Next, samples (EtOH solutions and EtOH solutions of $\mathrm{HgCl}_{2}$ ) were applied to cellulose TLC-plate, which was not activated. After drying with hair dryer for complete removal of solvent, we did not develop chromatographic layer in any chromatographic mobile phase. Next, the plate was immediately sprayed with enzyme solution and it was incubated at RT for $15 \mathrm{~min}$. Finally, the NADPH/NBT/PMS staining solution was sprayed onto TLC plate. Approximately $3.5 \mathrm{ml}$ of staining solution was distributed over $5 \times 5 \mathrm{~cm}$ TLC plate. Final reagents concentrations in this volume were as follows: $35 \mathrm{mM}$ TRIS/ $\mathrm{HCl}$ buffer $\mathrm{pH} 7.65,7 \mathrm{mM} \mathrm{mgCl}_{2} \times$ $6 \mathrm{H}_{2} \mathrm{O}, 0.35 \mathrm{mM}$ fru-6P, $12 \mathrm{U}$ glc-6P dehydrogenase from $L$. mesenteroides, $1 \mathrm{mg} \mathrm{NADP}+, 0.57 \mathrm{mg} \mathrm{NBT}, 0.057 \mathrm{mg}$ PMS and deionized water up to final volume.

After application of staining solution, the plate (closed in Petri dish) was placed in the incubator Innova ${ }^{\mathrm{TM}} 42$ (New Brunswick Scientific, USA) in the dark at $37^{\circ} \mathrm{C}$ for $20 \mathrm{~min}$. Clear yellow spots against a dark purple background indicated lack of PGI activity. A scheme presents (see Fig. 1) the lack of purple formazan formation from yellow NBT caused by the inhibition of PGI activity.

In order to confirm the lack of dehydrogenase glc-6P inhibition by $\mathrm{HgCl}_{2}$ there was performed analogous TLCautography as PGI. However, final reagents concentrations in this volume were as follows: $35 \mathrm{mM}$ TRIS/HCl buffer $\mathrm{pH} 7.65,7 \mathrm{mM} \mathrm{mgCl}_{2} \times 6 \mathrm{H}_{2} \mathrm{O}, 0.35 \mathrm{mM}$ glc-6P, $12 \mathrm{U}$ glc-6P dehydrogenase from $L$. mesenteroides, $1 \mathrm{mg} \mathrm{NADP}{ }^{+}$, $0.57 \mathrm{mg}$ NBT, $0.057 \mathrm{mg}$ PMS and deionized water up to final $(3.5 \mathrm{ml})$ volume.

\section{RESULTS AND DISCUSSION}

The challenge was to perform the bioassay for the detection E. coli ATCC 25922 PGI activity or inhibition of this activity directly on the surface of thin-layer plate.

Initial studies have indicated the fact that direct measurement of phosphoglucose isomerase activity from $E$. coli 


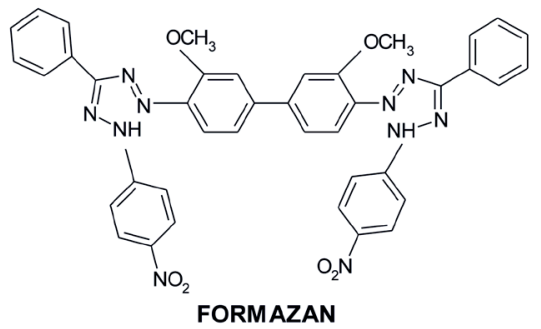

Inhibition of reaction catalyzed by $\mathbf{P G I}$

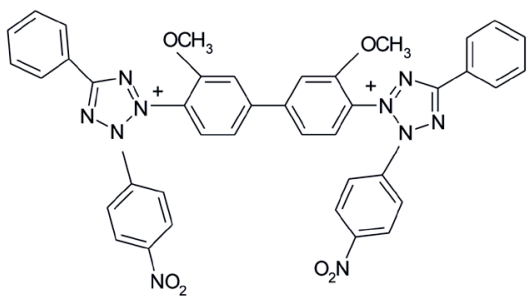

NBT

Figure 1. Blocking of purple formazan formation from yellow nitroblue terazolium chloride (NBT) coupled with the inhibition of phosphoglucose isomerase activity

ATCC 25922 on the glass-backed TLC layer of silica gel 60 $\mathrm{F}_{254}$ is not possible. It should be mentioned that an immobilization of characterized enzyme was tested. In order to find suitable method to detect enzyme activity on TLC-plate, a gel entrapment of E. coli ATCC 25922 PGI in 2\% agar or $1 \%$ agarose (both agents with low gelling temperature) distributed on the glass-backed TLC layer of silica gel 60 $\mathrm{F}_{254}$ was performed. These procedures were carried out with no success. Meanwhile, the known literature data demonstrated that gel entrapment of xanthine oxidase [22] and $\beta$-glucosidase [24] in agar after solidification on an aluminium-backed silica $60 \mathrm{~F}_{254}$ could be successfully used to search for inhibitors of both enzymes.

Also, investigation of PGI inhibition (in direction glc-6P to fru-6P) with Seliwanoff staining or PAHBAH staining under TLC-autography was unsuccessful (data not shown).

In the initial experiments, we wanted to check the possibility of enzymatic activity detection on cellulose plates by chemical procedures. For both tested types of arrays, fru-6P direction was detected as a final product of reaction catalyzed by PGI.

In Seliwanoff test, D-fructose is turned into orange/pink resorcinol-hydroxymethylfurfural complex, by treatment with resorcinol and boiling aqueous $\mathrm{HCl}$. Modification of this method proposed by Roe (use of alcoholic resorcinol solution, raised $\mathrm{HCl}$ concentration and heating at $80^{\circ} \mathrm{C}$ for $8 \mathrm{~min}$.) enabled the determination of both fructose and phosphate esters [23]. Therefore, we decided to apply Roe's modification for fru-6P staining on TLC-autography. However, incubation with Roe's solution $(0.1 \%$ alcoholic resorcinol and $30 \% \mathrm{HCl}$ ) did not produce an expected orange/pink color which confirmed that this type of staining was not effective for fru-6P development on TLC plates.
According to the earlier observations [3], PAHBAH can be successfully used for fru-6P identification as a chromogenic agent, producing yellow color. Thus, we performed TLC-autography of $E$. coli PGI using a staining solution containing $5 \%(\mathrm{w} / \mathrm{v})$ PAHBAH in $0.5 \mathrm{M} \mathrm{HCl}$, diluted directly before the experiment in $1: 4(\mathrm{v} / \mathrm{v})$ ratio in $0.5 \mathrm{M}$ $\mathrm{NaOH}$. After spraying TLC plate with the prepared staining PAHBAH solution, the autogram was heated at $100^{\circ} \mathrm{C}$ for $10 \mathrm{~min}$. As we did not observe a yellow color indicating the fru-6P as an end-product of PGI-catalyzed reaction, this approach failed to allow the screening of $E$. coli PGI activity during TLC-autography.

Both characterized assays are time-consuming. Moreover, Roe's method requires the use of hazardous chemicals.

Based on these premises we had to perform experiment with cellulose TLC-plate with NADPH/NBT/PMS staining for TLC-autographic analysis. In this model, we used $\mathrm{HgCl}_{2}$ as a noncompetitive inhibitor of $E$. coli PGI (Fig. 2). Parts 2.2 and 2.4 of autogram clearly showed that PGI-inhibitory action of $\mathrm{HgCl}_{2}$ was visualized by yellow spots on TLC plate, due to a lack of NBT conversion.
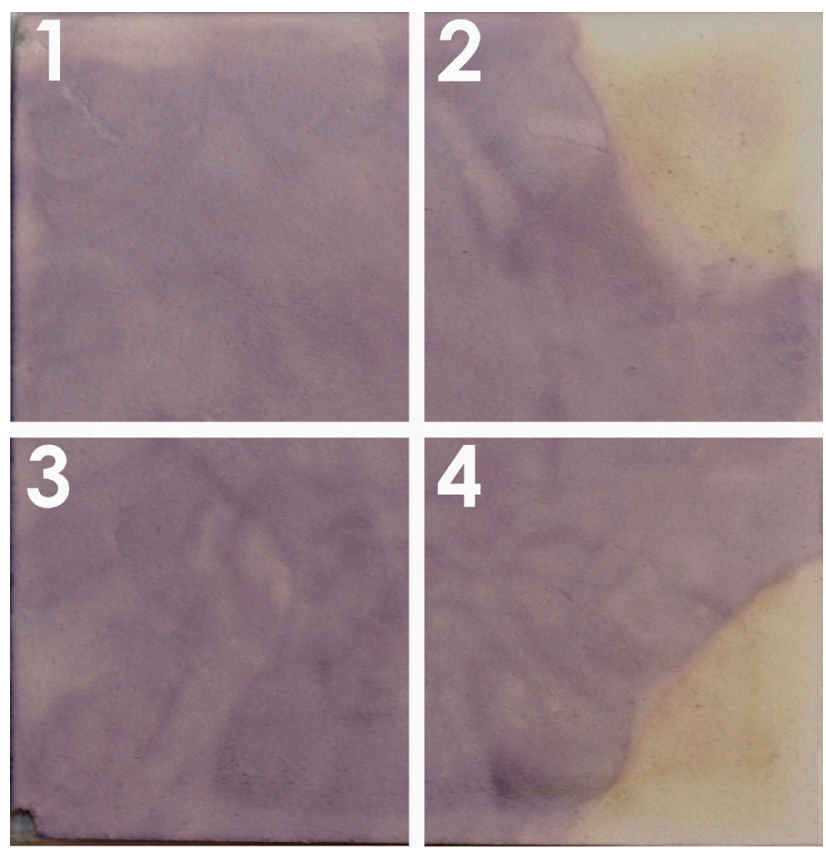

Figure 2. Autographic assay showing the inhibition of phosphoglucose isomerase by mercury(II) chloride using $\mathrm{NADP}^{+} / \mathrm{NBT} /$ PMS staining. The assay was carried out using cellulose TLC plate that was not eluted with mobile phase. For assay protocol, see Materials and Methods section. Phosphoglucose isomerase activity sprayed onto TLC plate was 0.079 U. Part $1-10 \%$ [v/v] $\mathrm{EtOH}$ in water; part $2-110 \mu \mathrm{g}$ of $\mathrm{HgCl}_{2}$ in $10 \%[\mathrm{v} / \mathrm{v}] \mathrm{EtOH}$ in water; part $3-25 \%[\mathrm{v} / \mathrm{v}] \mathrm{EtOH}$ in water and part $4-110 \mu \mathrm{g}$ of $\mathrm{HgCl}_{2}$ in $25 \%[\mathrm{v} / \mathrm{v}] \mathrm{EtOH}$ in water

According to the earlier study [3], heavy metal ions such as $\mathrm{Hg}(\mathrm{II})$ are considered as the major inhibitors of PGI catalyzed reaction. The main targets of mercury ions are sulfhydryl groups in e.g. cysteine - which is often localized in the active site of various enzymes. Mercury chloride binds to a single residue with forming R-S-Hg-Cl [9]. Also, this inorganic compound is able to cleave of S-S bridges. This results in disappearance of tertiary structure of biocatalyst and hence in the reduction of its stability [9]. 
The available information [11] reveals that cysteine is absent in the catalytic site of glc-6P dehydrogenase from L. mesenteroides. Nevertheless, a control experiment with respect to this enzyme was performed. Figure 3 displays the result of TLC-autography of glc-6P dehydrogenase with NADPH/NBT/PMS staining. One should notice that the incubation step of plate in the dark at $37^{\circ} \mathrm{C}$ had to be extended this time. As presented on $2^{\text {-nd }}$ and $4^{\text {th }}$ parts of this autogram, it lacks the yellow spots indicating inhibition zones of the auxiliary enzyme activity by mercury cations.
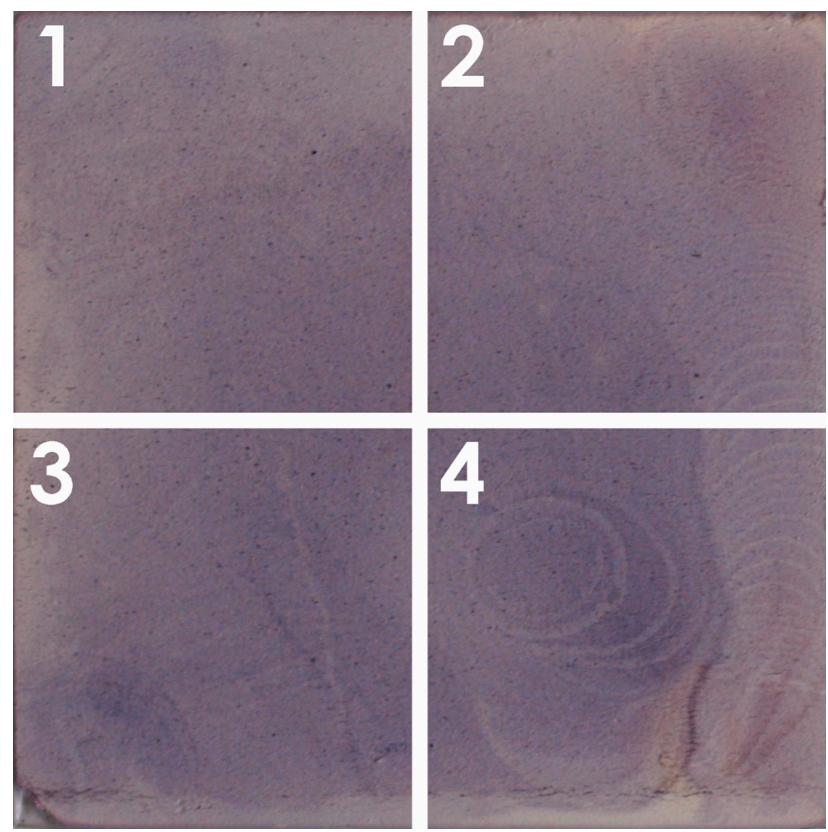

Figure 3. Autographic assay showing the lack inhibition of dehydrogenase glc-6P from $L$. mesenteroides by mercury(II) chloride using $\mathrm{NADP}^{+} / \mathrm{NBT} / \mathrm{PMS}$ staining. The assay was carried out using cellulose TLC plate that was not eluted with mobile phase. For assay protocol, see Materials and Methods section. Activity of dehydrogenase glc-6P sprayed onto TLC plate was 12 U. Part $1-10 \%$ [v/v] EtOH in water; Part $2-110 \mu \mathrm{g}$ of $\mathrm{HgCl}_{2}$ in $10 \%[\mathrm{v} / \mathrm{v}] \mathrm{EtOH}$ in water; Part $3-25 \%[\mathrm{v} / \mathrm{v}] \mathrm{EtOH}$ in water and Part $4-110 \mu \mathrm{g}$ of $\mathrm{HgCl}_{2}$ in $25 \%$ [v/v] EtOH in water

To our knowledge, we were the first to report TLC-autography as a technique to identify the inhibitors of phosphoglucose isomerase. However, further research regarding the modification of the solvent content and mobile phases may bring more satisfactory results. In our further study, we will focus on screening of plants as resources of inhibitors of PGI isolated from $E$. coli.

\section{ACKNOWLEDGEMENTS}

The paper was developed using the equipment purchased within the agreement No. POPW.01.03.00-06-010/09-00 Operational Program Development of Eastern Poland 20072013, Priority Axis I, Modern Economy, Operations 1.3. Innovations Promotion. The authors are grateful to Department of Physical Chemistry, Medical University of Lublin, Poland for providing Cellulose TLC layers.

\section{REFERENCES}

1. Adhami H-R., Farsam H. and Krenn L.: Screening of medicinal plants from Iranian traditional medicine for acetylcholinesterase inhibition. Phytother. Res., 25, 1148, 2011.
2. Adhami H-R. et al.: Compounds from Gum Ammoniacum with acetylcholinesterase inhibitory activity. Sci. Pharm., 81, 793, 2013.

3. Bednarz B.: Purification and characterization of phosphoglucose isomerase from E. coli ATCC 25922. Master's thesis. Medical University of Lublin, Poland, 2010.

4. Benamar H. et al.: Screening of Algerian medicinal plants for acetylcholinesterase inhibitory activity. J. Biol. Sci., 10, 1, 2010.

5. Choma I.M. and Grzelak E.M.: Bioautography detection in thin-layer chromatography. J. Chromatogr. A., 1218, 2684, 2011.

6. Dewanjee S. et al.: Bioautography and its scope in the field of natural product chemistry. J. Pharm. Anal., http://dx.doi.org/10.1016/j. jpha.2014.06.002.

7. Ebrahim N. and Uebel R.A.: Direct inhibition of cyclooxygenase-2 enzyme by an extract of Harpagophytum procumbens, harpagoside and harpagide. AJPP., 5, 2209, 2011.

8. Favre-Godal Q., Queiroz E.M., Wolfender J.-L.: Latest development in assessing antifungal activity using TLC-bioautography: A review. J. AOAC Int., 96, 1175, 2013.

9. Frasco M.F. et al.: Mechanism of cholinesterase inhibition by inorganic mercury. FEBS. J., 274, 1849, 2007.

10. Hassan A.M.S.: TLC bioautographic method for detecting lipase inhibitors. Phytochem. Anal., 23, 405, 2012.

11. Ishaque A., Milhausen M. and Levy R.: On the absence of cysteine in glucose 6-phosphate dehydrogenase from Leuconostoc mesenteroides. Biochem. Biophys. Res. Commun., 59, 894, 1974.

12. Liang J.B., Yang Z.D., Shu Z.M. and Yu C.C.: A rapid thin-layer chromatography bioautographic method for detecting the monoamine oxidase inhibitors in plants. Nat. Proc. Res., 28, 1318, 2014.

13. Maninang J.S., Lizada M.C.C. and Gemma H.: Inhibition of aldehyde dehydrogenase enzyme by Durian (Durio zibethinus Murray) fruit extract. Food Chem., 117, 352, 2009.

14. Marston A., Kissling J. and Hostettmann K.: A rapid TLC bioautographic method for the detection of acetylcholinesterase and butyrylcholinesterase inhibitors in plants. Phytochem. Anal., 13, 51, 2002.

15. Marston A.: Thin-layer chromatography with biological detection in phytochemistry. J. Chromatogr. A,1218, 2676, 2011.

16. Mehrabani M. and al.: Evaluation of antifungal activities of Myrtus communis L. by bioautography method. Jundishapur J. Microbiol., 6, e8316, 2013.

17. Móricz Á.M., Tyihák E. and Ott P.G.: Usefulness of transgenic luminescent bacteria in direct bioautographic investigation of chamomile extracts. J. Planar Chromatogr., 23, 180, 2010.

18. Mroczek T. and Mazurek J.: Pressurized liquid extraction and anticholinesterase activity-based thin-layer chromatography with bioautography of Amaryllidaceae alkaloids. Anal. Chim. Acta, 633, 188, 2009.

19. Pandey S. et al.: A novel method for screening beta-glucosidase inhibitors. BMC Microbiol., 13, 55, 2013.

20. Paradowska K., Bednarz B. and Ginalska G.: Phosphoglucose isomerase from Escherichia coli ATCC 25922 - pilot studies. Annales UMCS, Sect. DDD, XXIII, 87, 2010.

21. Patil N.N. et al.: Bioautography quided screening of antimicrobial compounds produced by Microbispora V2. Int. Res. J. Biological Sci., $2,65,2013$.

22. Ramallo A., Zacchino S.A. and Furlan R.L.E.: A rapid TLC autographic method for the detection of xanthine oxidase inhibitors and superoxide scavengers. Phytochem. Anal., 17, 15, 2006.

23. Roe J.H. and Papadopoulos N.M.: The determination of fructose6-phosphate and fructose-1,6-diphosphate. J. Biol. Chem., 210, 703, 1954.

24. Salazar M.O. and Furlan R.L.E.: A rapid TLC autographic method for the detection of glucosidase inhibitors. Phytochem. Anal., 18, 209, 2007.

25. Simões-Pires C.A. et al.: A TLC bioautographic method for the detection of $\alpha$ - and $\beta$-glucosidase inhibitors in plant extracts. Phytochem. Anal., 20, 511, 2009.

26. Warburg O. and Christian W.: Isolation and crystallization of enolase. Biochem. Z, 310, 384, 1942. 